\title{
DESENVOLVIMENTO DE UM TORQUIMETRO PARA MEDIÇÃO DINÂMICA DE MICROTORQUE
}

\author{
Pascoato R. ; Aranha L. C.; Mucsi C. S.; Rossi J. L. \\ Instituto de Pesquisa Energéticas e Nucleares - IPEN, São Paulo
}

Av. Prof. Lineu Prestes, 2242, Cidade Universitária - CEP 05508-00, São Paulo, SP, Brasil

*e-mail: rpascoato@uol.com.br

\begin{abstract}
Resumo
Foi desenvolvido um dispositivo protótipo de um torquímetro para ensaio de medição dinâmica de micro torques. Este dispositivo foi usado para a avaliação de implantes ortodônticos. O ensaio consistiu na inserção e retirada de pilares protéticos em implantes odontológicos, com torques menores que $100 \mathrm{Ncm}$. O principal componente desse protótipo foi a barra de torque, nesta, foi aplicado um extensômetro tipo strain gage. As deformações sofridas por este componente, não descriminavam entre deformações devido a tensões de flexão e tensões de torção. Com o propósito de diminuir esta sensibilidade a deformação por flexão e aumentar a sensibilidade da torção, foi proposta uma nova geometria para a barra de torque. Após ser estudada analiticamente, esta nova geometria mostrou- se bastante favorável quanto ao aumento da sensibilidade à torção do componente e a diminuição da interferência devido ao seu peso próprio.
\end{abstract}

\begin{abstract}
It was developed a torque wrench device to measure the dynamic of the micro torque. This device was used to check orthodontic implants. The test consisted in the insertion and removal of abutments for dental implants with torques less than $100 \mathrm{Ncm}$. The main component of this device was the torque bar, it was applied a type of extensometer strain gage. The deformations applied on this component did not discriminated between strains due to bending stresses and torsional stresses. In order to decrease this sensitivity to bending deformation and increase the sensitivity of torque, it was proposed a new geometry for the torque bar. After analyticall Studies, this new geometry was shown an increase on the sensitivity to torsional component and decrease the interference due to its own weight.
\end{abstract}

Palavras chaves: medição dinâmica de micro torques, extensômetria, implantes e componentes protéticos

\section{Introdução}

Para que se mantenha a integridade do sistema de implantes ortodônticos, é necessário seu correto entendimento. Caso haja a necessidade de troca do componente protético, este não deve sofrer deformações [1].

$\mathrm{Na}$ inserção dos implantes dentários os torques de inserção devem se restringir a valores baixos, para evitar danos ao osso. Através da experiência prévia foi observados que altos torques de inserção podem atrapalhar o processo cicatricial. Se altos torques forem utilizados no processo de implantação ocorre o aquecimento local gerando desidratação das células e micro fraturas de osso medular que têm, como consequência, a necrose óssea e falha de cicatrização.
O desempenho do implante dentário depende em especial da estabilidade desse dispositivo durante o processo de cicatrização, denominado de osseointegração. Afirma-se, na literatura [2,3] que para atingir essa tão importante estabilidade, é fundamental o formato do implante e a técnica cirúrgica a ser utilizada. Na falta dessa estabilidade ocorre a formação de uma cápsula de tecido mole ao redor do implante, prejudicando a sustentação do dente que receberá uma alta carga durante a mastigação.

Já na fase protética, após a osseointegração, um pilar é fixado através de roscas sobre o implante. Este receberá o dente artificial ou prótese dental. Nesta fase o torque também deve ser preciso no intuito de se manter o menor espaço entre as peças sem que ocorra qualquer tipo de deformação plástica, nesse espaço podem ser retidas colônias de bactérias que se tornam 
fatores de agressão ao osso adjacente ao implante. Isto pode causar reabsorção do osso ao redor do implante prejudicando a estética e a função.

Devido à indisponibilidade de um equipamento para a medida de micro torques, com a sensibilidade adequada e a taxa de aquisição de dados requerida, foi desenvolvido um protótipo de dispositivo para a medição de micro torque, para a inserção e retirada de elementos roscados, como os utilizados na área de implantes dentários. Isto com o objetivo de oferecer informações precisas para a elaboração de protocolos de aplicação de do sistema protético, visando garantir a integridade da ancoragem e estabilidade deste sistema (prótese e pilar) [4]. Na Figura 1 é apresentada uma foto do protótipo

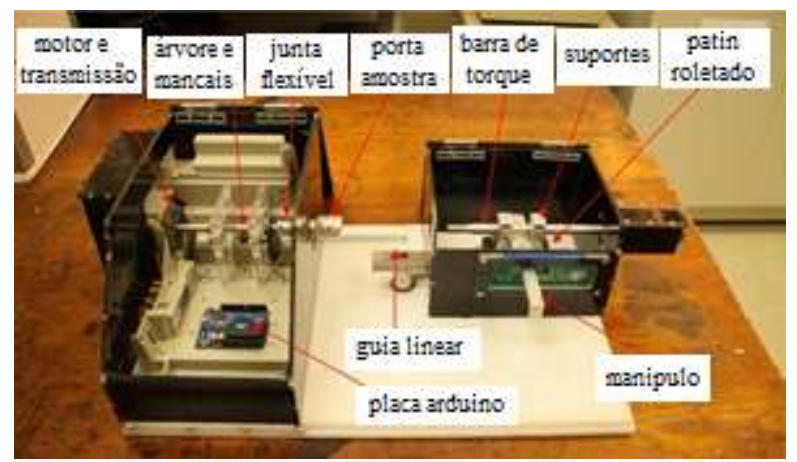

Figura 1: Protótipo do dispositivo para a medição dinâmica de micro torques desenvolvida no CCTM - IPEN.

\section{Objetivos}

Investigar, inicialmente, por meio de cálculos de engenharia, os esforços mecânicos a que são submetidos os componentes de um dispositivo de medição de micro torque de inserção de próteses e pilares dentários. Após esta etapa do trabalho, devemse propor alterações do projeto com o objetivo de eliminar a presença de esforços de flexão, mantendo o extensômetro, do tipo strain gauge, exclusivamente sujeito à cargas de torção, dentro dos limites de operação estabelecidos.

Preliminarmente pretende-se aumentar a resolução de medida do aparelho em 3 vezes, através do aumento da flexibilidade da barra de torque, do dispositivo para a medição de micro torque, seja pela substituição do material de construção ou seja pelo reprojeto da geometria da barra de torque.

\section{Materiais e métodos}

Na Figura 2 é apresentada uma imagem da barra de torque inicialmente utilizada. Este componente é parte fundamental do dispositivo de torque mostrado na imagem da Figura 1. Sobre a barra de torque é colado o extensômetro do tipo strain gauge, que serve de transdutor [5]

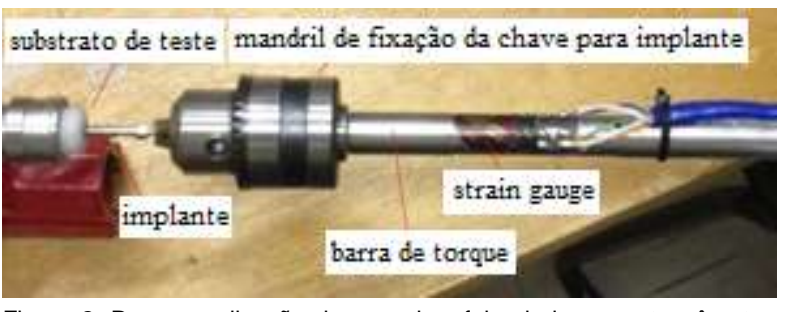

Figura 2: Para a realização dos ensaios, foi colado um extensômetro, do tipo strain gauge, na barra de torque.

Foram calculados os esforços de flexão e de torção a que a barra de torque é submetida, seja pelo peso do próprio equipamento ou pelos esforços da operação. Os esforços aplicados à barra torque são mostrados na Figura 3.

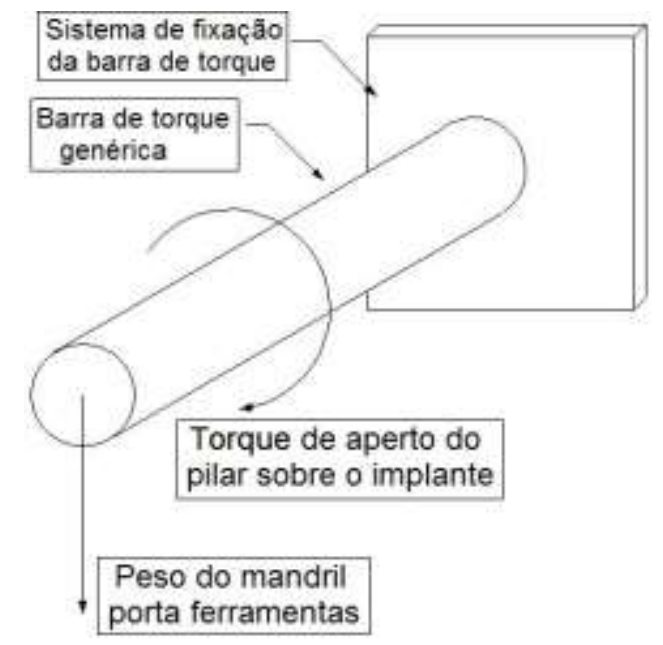

Figura 3: Desenho esquemático de barra de torque genérica, mostrando os esforços a que a barra é submetida.

O extensômetro, aplicado sobre a superfície da barra de torque, não discrimina a origem de suas deformações, sejam elas de torção ou de flexão; assim, o peso do mandril porta ferramentas pode ter um efeito sobre a deformação do extensômetro tão importante quanto o torque aplicado sobre o mesmo. Em função deste comportamento serão determinadas e comparadas as deformações devidas à flexão e à torção na barra de torque original e no novo modelo proposto.

\section{Resultados e discussão}

Para atingir os objetivos propostos para este trabalho, à barra de torque do protótipo do dispositivo foi analisada, quanto às suas resistências à flexão, causada pelo peso do mandril e pelo seu peso próprio e quanto à torção, causada pelos esforços de torqueamento. Assim, foram analisadas as propriedades de resistência mecânica devida à geometria de um componente sujeito a esforços mecânicos depende da sua seção transversal.

Foi concebida uma nova geometria para a barra de torque, aumentando a sua resistência à flexão vertical e 
a sua deformação angular. Esta nova concepção de geometria é apresentada na Figura 4. Novamente, foi calculada a resistência à torção, para, a seguir, ser comparada àquela do projeto original. [6]

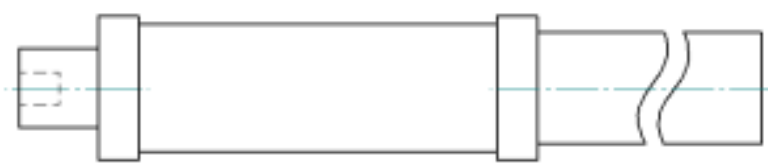

Figura 4: Desenho representativo da nova geometria da barra de torque.

Foram obtidas analiticamente as deformações devidas aos esforços de torção e flexão, de operação do equipamento, para a geometria atual, Tabela 1, e para a nova geometria proposta, Tabela 2.

Tabela 1: Deformações de torção e flexão, sofridas pela barra de torque com geometria atual.

\begin{tabular}{ccccc} 
ø ext. & $\varnothing$ int. & Comp. & Torção & Flexão \\
\hline $1,100 \mathrm{~cm}$ & $0,892 \mathrm{~cm}$ & $1,5 \mathrm{~cm}$ & $0,084053^{\circ}$ & $2,4 \times 10^{-6} \mathrm{~mm}$ \\
\hline
\end{tabular}

Tabela 2: Deformações sofridas pela barra de tor-que com nova geometria proposta.

\begin{tabular}{cccc}
\hline Espessura & Comprimento & Torção & Flexão \\
\hline $0,300 \mathrm{~cm}$ & $1,5 \mathrm{~cm}$ & $0,142270^{\circ}$ & $2,1 \times 10^{-7} \mathrm{~mm}$ \\
\hline
\end{tabular}

Os resultados obtidos, para a torção de uma lâmina com espessura de $3 \mathrm{~mm}$, a deformação angular igualase a 2,5 vezes aquela da deformação da barra de torque original. Para a flexão dessa mesma lâmina, obteve-se um aumento da resistência a flexão de 11 vezes em relação com a barra de torque original.

\section{Mockup}

Na Figura 5, mostra uma imagem dos primeiros testes do mockup plástico montado no protótipo.

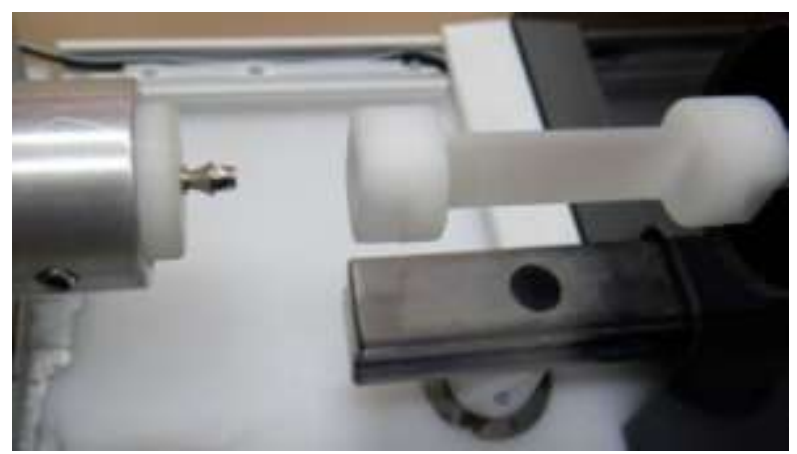

Figura 5: Mockup da barra de torque com nova geometria proposta, montado no protótipo atual do micro torquímetro.

\section{Conclusão}

O protótipo atual exige que seja dada atenção aos esforços de flexão na barra de torque.
A nova geometria proposta para a barra de torque permitirá que as medidas sejam realizadas sem a interferência de esforços de flexão.

A maior deformação projetada, para um mesmo esforço de torção, permitirá a leitura de menores valores de torque, com a mesma sensibilidade do sistema atual.

O reprojeto das partes críticas do equipamento permitirá a construção de um torquímetro com maiores resolução, sensibilidade, repetibilidade, precisão e exatidão de medida; não necessitando das constantes calibrações atualmente realizadas.

\section{Agradecimentos}

Os autores agradecem a CAPES pela bolsa de estudos para Pascoato, $\mathrm{R}$.

\section{Referências}

[1] ARANHA, L.C. Estudo dos parâmetros de estabilidade de travamento entre implante dentário e componente protético através do sistema cone morse. 2014. 89 f. Tese (Doutorado em Tecnologia Nuclear Materiais) - Instituto de Pesquisas Energéticas e Nucleares, Universidade de São Paulo, São Paulo. 2014.

[2] LIOUBAVINA-HACK, N.; LANG, N.P.; KARRING, T. Significance of primary stability for osseointegration of dental implants. Clin. Oral Implants Res. 2006;17:24450 .

[3] BAREWAL, R.M.; OATES, T.W.; MEREDITH, N.; COCHRAN, D.L. Resonance frequency measurement of implant stability in vivo on implants with a sandblasted and acid-etched surface. Int. J. Oral Maxillofac Implants. 2003; 18(5):641-51.

[4] MUCSI, C.S.; NOGUEIRA E. J.; ARANHA, L. C.; CEONI, F.C. ; ROSSI, J.L. Desenvolvimentos de tecnologia e dispositivo para a medição de torque de fixação de elementos roscados. $5^{\circ}$ Congresso Científico da Semana Tecnológica - IFSP. 2014, 20-24 de outubro de 2014, Bragança Paulista, SP, Brasil.

[5] CEONI, F.C.; ROSSI, J.L.; SOUZA, A.C.; COLOSIO, M.A. Residual stresses measurements using strain gages -aluminum wheels. Materials Science Forum. 2012;727-728:1925-1930.

[6] PASCOATO, R.; NOGUEIRA, E.J ARANHA, L.C.; MUCSI, C.S.; ROSSI, J.L. Projeto mecânico de componentes, para medição precisa de micro torques em dispositivo de avaliação de implantes dentários. 6은 Congresso Científico da Semana Nacional de Ciência e Tecnologia - IFSP. 20-23 de outubro de 2015 - Instituto Federal de São Paulo Bragança Paulista, SP, Brasil. 